\title{
Induced orientational order of anisotropic nanoparticles in the lamellae phase of diblock copolymers
}

\author{
M. A. Osipov ${ }^{1,2}$, M. V. Gorkunov ${ }^{3}$, and Y. V. Kudryavtsev ${ }^{2}$ \\ 1 Department of Mathematics and Statistics, \\ University of Strathclyde, Glasgow G1 1 XH, Scotland, UK \\ 2 Topchiev Institute of Petrochemical Synthesis, \\ Russian Academy of Sciences, 119991 Moscow, Russia \\ 3 Shubnikov Institute of Crystallography of Federal Scientific Research Centre \\ "Crystallography and Photonics" of Russian Academy of Sciences, 119333 Moscow, Russia
}

(Dated: November 11, 2016)

\begin{abstract}
Orientational order and spatial distribution of anisotropic nanoparticles in the lamellae phase of block copolymers have been considered in the case of strong segregation taking into account both isotropic and anisotropic interaction between nanoparticles and the polymer chains. Explicit analytical results have been obtained for the distribution of anisotropic nanoparticles in the lamellae phase and the nematic order parameter profiles have been calculated numerically. It has been shown that anisotropic nano-paticles are orientationally ordered in the boundary region between the blocks and the nematic order parameter possess opposite signs in different blocks.
\end{abstract}

PACS numbers: nanocomposites, orientational order, anisotropic interaction 


\section{INTRODUCTION}

At present a significant attention is attracted to composite LC and polymer materials doped with various nanoparticles. Polymer nanomaterials that contain metal, oxide, silicate or semiconductor nanoparticles have a number of advantages over pure polymer systems as the introduction of nanoparticles improves various characteristics of polymer materials [? ? ? ? ? ? ? ]. Microphase separation in block copolymers can also stabilize the spatially inhomogeneous distribution of the nanoparticles, which in principle enables one to develop highly ordered materials with controlled parameters. Such materials can be used as novel membranes and employed in nanophotonics [? ? ]. In particular, recent studies of nanorods (NRs) in homopolymer [? ? ? ? ] show that dispersion and alignment can result in a tunable structure-property relationship. The use of anisotropic nanoparticles opens an exciting possibility to align the lamellae and hexagonal phases by external fields which may help to solve some application problems as there exist no simple and reliable methods to alignment block copolymers.

With few exceptions (see, for example,[? ? ? ? ]) the existing theoretical studies of polymer nanocomposites are confined to the case of isotropic nanoparticles [? ]. So far there has been no systematic investigations of the effect of nanoparticle anisotropy on the phase behavior of diblock copolymers and of the effect microphase separation on the orientational and translational ordering of anisotropic nanoparticles. . At the same time, the anisotropy of nanoparticles is known to play a significant role in the related soft matter systems. In particular, it has been shown that anisotropic composites composed of low molecular weight liquid crystals (LC) doped with nanoparticles possess a number of improved characteristics in comparison with conventional LCs including lower threshold voltages and the switching times of LC displays [? ? ? ? ? ]. The introduction of nanoparticles in various LC materials enables one to extend the range of several LC phases, to raise the temperature of phase transitions and to improve the conditions of lasing in the LC matrix doped with dyes [? ? ? ? ? ]. Recently a molecular theory has been developed to explain the effect of nanoparticles on thermodynamic and dielectric properties of low molecular weight liquid crystals [? ? ? ? ? ] One expects that composites based on LC polymers and anisotropic nanoparticles will offer a number of benefits over the conventional LC polymers.

There exists an experimental evidence that anisotropic nanoparticles may be aligned 
in lamellae and hexagonal phases of block copolymers even at very low concentrations.For example it has been found that poly(ethylene glycol) (PEG) functionalized gold nanorods are aligned parallel to the lamellae planes [? ]. At the same time the polystyrene functionalized nano-rods are orientationally ordered in the hexagonal phase of the same block copolymer with their long axes perpendicular to the cylinders[? ? ]. In contrast, the alkyl phosphonic acid capped nano-rods are ordered parallel to the cylinders in a different block copolymer $[?]$.

The effect of nanoparticles on the properties of block copolymers is twofold. On the one hand the nanoparticles may effect the relative stability of different phases and shift the corresponding transition temperatures. On the other hand, the microphase separation in the polymer matrix may induce inhomogeneous periodic distribution of nano-particles which. in turn, may effect dielectric, optical and mechanical properties of block copolymers. In this paper we address the second topic, and calculate the spatial distribution of anisotropic nanoparticles and the orientational order parameter profiles in the lamellae phase, taking into account both isotropic and anisotropic interactions between nano-particles and monomers.

The most interesting effects are predicted to exist in the vicinity of the interface between the blocks. In this region the anisotropic nano-particle may interact simultaneously with different monomers located in adjacent blocks. This asymmetric interaction generally induces orientational order of anisotropic nano-particles in the interfacial region/ Moreover, nanoparticles may align parallel and perpendicular to the interface on different sides of the boundary. In the hexagonal phase the curvature of the cylindrical interface also induces the biaxial order of nano-particles.

\section{LAMELLAR PHASE. STRONG SEGREGATION}

Let us consider the idealised diblock-copolymer with fixed density distribution of $\mathrm{A}$ and B segments doped with nanoparticles.. In the simplest lamellar phase the total periodicity is $d=d_{A}+d_{B}$ where $d_{A}$ is the thickness of the A-rich layer and $d_{B}$ is that of the B-rich layer, respectively.

Let us first consider the ideal copolymer in the strong separation limit. In this case, the block $\mathrm{A}$ is composed only of monomers $\mathrm{A}$ while the block $\mathrm{B}$ is composed of monomers B. Then the average number density of monomers $\mathrm{A}$ is expressed as $\rho_{A}(\mathbf{r})=\rho_{0 A}$ when 
$0<z<d_{A}, \rho_{A}(\mathbf{r})=0$ when $d_{A}<z<d$ and $\rho_{A}(z)=\rho_{A}(z+d)$ for all $z$ as it is a periodic function with the period $d$. The average number density of monomers $\mathrm{B}$ is given by a similar expression : $\rho_{B}(\mathbf{r})=\rho_{0 B}$ when $d_{A}<z<d, \rho_{B}(\mathbf{r})=0$ when $0<z<d_{A}$ and $\rho_{B}(z)=\rho_{B}(z+d)$ for all $z$.

We will use a very simple expression for the interaction potential between the nanoparticle $i$ and the monomers which is composed of the isotropic and anisotropic parts:

$$
U_{i}=\sum_{l=l_{A}}\left[J_{A}\left(r_{i l}\right)+I_{A}\left(r_{i l}\right)\left(\mathbf{a}_{i} \cdot \mathbf{u}_{i l}\right)^{2}\right]+\sum_{l=l_{B}}\left[J_{B}\left(r_{i l}\right)+I_{B}\left(r_{i l}\right)\left(\mathbf{a}_{i} \cdot \mathbf{u}_{i l}\right)^{2}\right],
$$

where $\mathbf{r}_{i}$ is the position vector of the nanoparticle $i$ and $\mathbf{a}_{i}$ is the unit vector in the direction of the long axis of the rod-like nanoparticle. Here $J_{A}\left(r_{i l}\right), I_{A}\left(r_{i l}\right)$ and $J_{B}\left(r_{i l}\right), I_{B}\left(r_{i l}\right)$ are the isotropic and anisotropic coupling constants between the nanoparticle and the monomers $\mathrm{A}$ and $\mathrm{B}$, respectively, $\mathbf{r}_{l A}$ and $\mathbf{r}_{l B}$ are the position vectors of the monomers $\mathrm{A}$ and $\mathrm{B}$, respectively, $\mathbf{r}_{i l}=\mathbf{r}_{i}-\mathbf{r}_{l}$ and $\mathbf{u}_{i l}$ is the unit vector in the direction of $\mathbf{r}_{i l}$.

The anisotropic interaction between isotropic monomers and anisotropic nano-particles in Eq. (??) describes the coupling between the long axis of a nanoparticle $\mathbf{a}_{i}$ and the unit vector $\mathbf{u}_{i l}$ pointing from the particle to the monomer.

In the molecular field approximation the one-particle distribution function of nanoparticles is given by the Boltzman distribution:

$$
f\left(\mathbf{a}_{i}, \mathbf{r}_{i}\right)=Z^{-1} \exp \left[-U_{M F}\left(\mathbf{a}_{i}, \mathbf{r}_{i}\right) / k_{B} T\right],
$$

where $Z$ is the normalization factor and the mean-field potential $U_{M F}\left(\mathbf{a}_{i}, \mathbf{r}_{i}\right)$ is expressed as:

$$
\begin{aligned}
U_{M F}\left(\mathbf{a}_{i}, \mathbf{r}_{i}\right)=\int\left[J_{A}\left(r_{i l}\right)+I_{A}\left(r_{i l}\right)\left(\mathbf{a}_{i} \cdot \mathbf{u}_{i l}\right)^{2}\right] & \rho_{A}\left(\mathbf{r}_{l}\right) d^{3} \mathbf{r}_{l}+ \\
& \int\left[J_{B}\left(r_{i l}\right)+I_{B}\left(r_{i l}\right)\left(\mathbf{a}_{i} \cdot \mathbf{u}_{i l}\right)^{2}\right] \rho_{B}\left(\mathbf{r}_{l}\right) d^{3} \mathbf{r}_{l} .
\end{aligned}
$$

The local scalar nematic order parameter of the nanoparticles with respect to the lamella normal $\mathbf{k}$ is then expressed as

$$
S(\mathbf{r})=\left\langle P_{2}\left(\mathbf{a}_{i} \cdot \mathbf{k}\right)\right\rangle=\frac{\int P_{2}\left(\mathbf{a}_{i} \cdot \mathbf{k}\right) f\left(\mathbf{a}_{i}, \mathbf{r}\right) d^{2} \mathbf{a}_{i}}{\int f\left(\mathbf{a}_{i}, \mathbf{r}\right) d^{2} \mathbf{a}_{i}},
$$

while the average density distribution if nano-particles is given by

$$
\rho_{N}(\mathbf{r})=\rho_{N 0} \int f\left(\mathbf{a}_{i}, \mathbf{r}\right) d^{2} \mathbf{a}_{i},
$$


where $\rho_{N 0}$ is the average nano-particle number density and $P_{2}(x)$ is the second Legendre polynomial.

The microscopic origin of the anisotropic interaction that couples the intermolecular vector with the primary axis of one of the particles can be illustrated in the following way.Firstly, it appears naturally in the expansion of practically any model interaction potential which depends on the molecular orientation and on the intermolecular vector [? ].Secondly, the corresponding term is present, for example, in the general expression for the dipole-dipole dispersion interaction potential [? ] One notes that a coupling between the intermolecular vector and the primary axis of one of the interacting molecules is usually not taken into account in the molecular theory of nematic liquid crystals because it is averaged out in the bulk in the molecular-field approximation. At the same time it is taken into consideration in the molecular theory of smectics C [? ] where it contributes to the free energy due to the existence of translational order. In block copolymers such an anisotropic interaction makes a contribution when a NP is close to the interface between the blocks, and it is in this region where the anisotropic Nps may be orientationally ordered.

The distribution of the nanoparticles can be determined only within a particular model for the interaction coupling constants. Let us assume for simplicity that $J_{\alpha}(r)=J_{\alpha} r^{-6}$ and $I_{\alpha}(r)=I_{\alpha} r^{-6}$, where $\alpha=A, B$, and there is a cut-off at small $r=r_{0}$, where $r_{0}$ is the nanoparticle radius. At larger $r$, there is another cut-off at $r=R_{0}$, where the $R_{0}$ is the range of interaction, which is assumed to be relatively small, $R_{0}<d_{A} / 2, d_{B} / 2$.

In this case some of the integrals in Eq. (??) can be taken analytically. Let us consider the mean-field potential $U_{M F}(\mathbf{a}, \mathbf{r})$ acting on a nanoparticle at some point $\mathbf{r}$. Taking into account that the interaction range is smaller than the half-widths of the blocks, one concludes that the nanoparticle at point $\mathbf{r}$ interacts with monomers inside the same block and with monomers of the other type in the closest adjacent block if the nanoparticle is sufficiently close to the boundary between the blocks. We denote by $L$ the distance from the nanoparticle to the closest boundary between the blocks.

Then the mean-field potential (??) acting on a particle in block A can be rewritten as:

$$
\begin{array}{r}
U_{M F}=\frac{1}{2} \int_{r_{0}<r<R_{0}} d V\left[\left(\rho_{0 A} J_{A}+\rho_{0 B} J_{B}\right)+\left(\rho_{0 A} I_{A}+\rho_{0 B} I_{B}\right)(\mathbf{a} \cdot \mathbf{u})^{2}\right] r^{-6}- \\
\int_{V_{0}} d V\left[\Delta J+\Delta I(\mathbf{a} \cdot \mathbf{u})^{2}\right] r^{-6},
\end{array}
$$



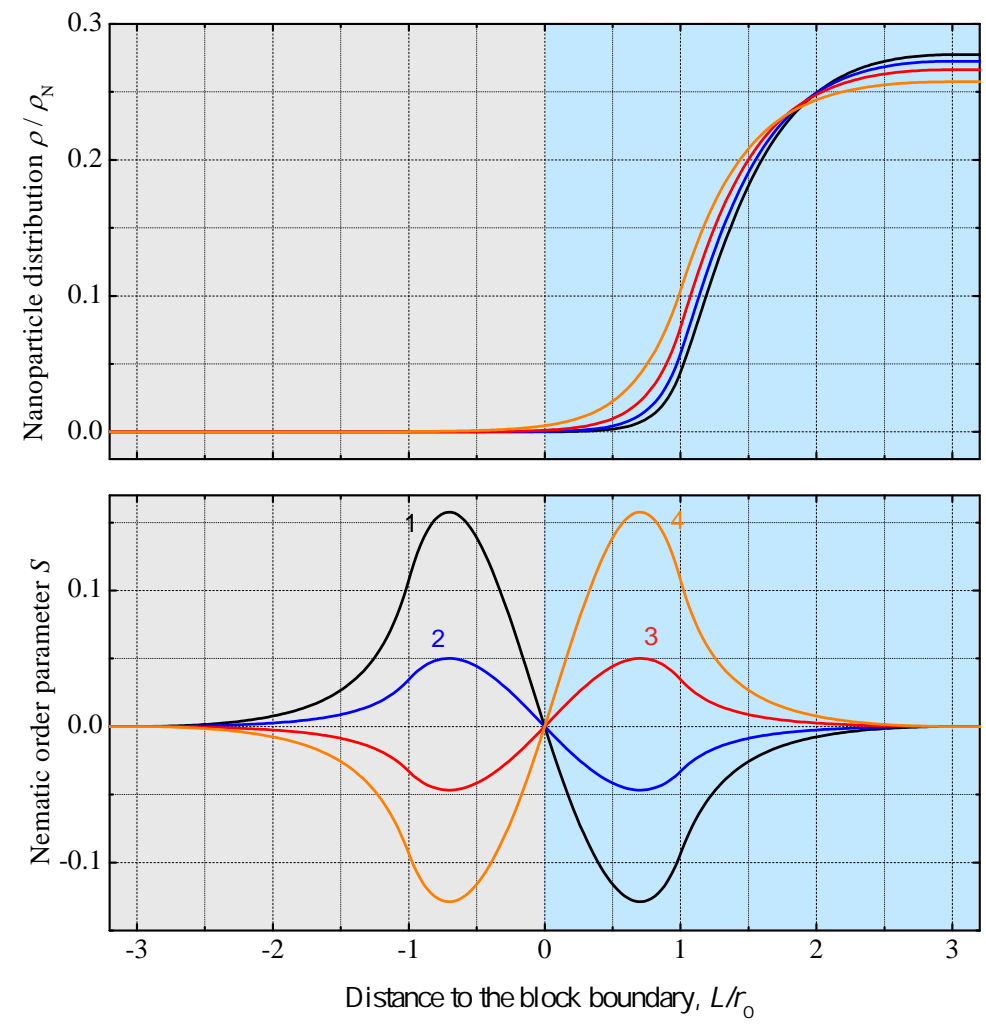

FIG. 1: Nanoparticle local density and nematic order parameter inside a lamellar diblock copolymer with strong segregation. The block thickness is $d_{A}=d_{B}=10 r_{0}$, the interaction radius $R_{0}=3 r_{0}$, the isotropic interaction constant $\Delta J=3 k_{B} T$ and the anisotropic constant $\Delta I=3 k_{B} T, k_{B} T,-k_{B} T,-3 k_{B} T$ for the lines from 1 to 4 respectively.

For a particle centered in block $\mathrm{B}$, one has to reverse the sign of the last term. Here the differences of the interaction parameters are introduced as $\Delta J=\rho_{0 B} J_{B}-\rho_{0 A} J_{A}$, and $\Delta I=$ $\rho_{0 B} I_{B}-\rho_{0 A} I_{A}$. The first integral is taken over the volume of the whole interaction sphere while the second one is taken over its fragment $V_{0}$ confined between the block boundary and the plane which contains the centre of the paprticle and which is parallel to the block boundary. The first term in Eq. (??) yields a constant independent of a and $L$, which can be neglected. The rest of the mean-field potential can be evaluated in a spherical coordinate system with the polar $z$-axis along the normal to the block boundary $\mathbf{k}$, the azimuthal angle $\phi$, and the polar angle $\theta$ measured from the $z$ axis. Then $\mathbf{u}=\mathbf{k} \cos \theta+\mathbf{x} \sin \theta \cos \phi+$ $\mathbf{y} \sin \theta \sin \phi$. 
For $L>R_{0}$ the potential reads as

$$
\begin{gathered}
U_{M F}^{(0)}(\mathbf{a}, L)=-\int_{r_{0}}^{R_{0}} r^{-4} d r \int_{0}^{1} d \cos \theta \int_{0}^{2 \pi} d \phi\left[\Delta+\Delta I(\mathbf{a} \cdot \mathbf{u})^{2}\right]= \\
=-2 \pi \int_{r_{0}}^{R_{0}} r^{-4} d r \int_{0}^{1} d \cos \theta\left[\Delta J+\frac{1}{2} \Delta I \sin ^{2} \theta+\Delta I P_{2}(\cos \theta)(\mathbf{k} \cdot \mathbf{a})^{2}\right]= \\
=\frac{2 \pi}{3}\left(\Delta J+\frac{\Delta I}{3}\right)\left(R_{0}^{-3}-r_{0}^{-3}\right),
\end{gathered}
$$

For an intermediate $r_{0}<L<R_{0}$, it takes the form

$$
\begin{gathered}
U_{M F}^{(1)}(\mathbf{a}, L)=-\left[\int_{r_{0}}^{L} d r \int_{0}^{1} d \cos \theta+\int_{L}^{R_{0}} d r \int_{0}^{L / r} d \cos \theta\right] \\
2 \pi r^{-4}\left[\Delta J+\frac{1}{2} \Delta I \sin ^{2} \theta+\Delta I P_{2}(\cos \theta)(\mathbf{k} \cdot \mathbf{a})^{2}\right]= \\
=\frac{2 \pi}{3}\left(\Delta J+\frac{\Delta I}{3}\right)\left(L^{-3}-r_{0}^{-3}\right)+ \\
+\frac{\pi L}{2}\left[\Delta J+\frac{\Delta I}{3}-\frac{\Delta I}{3} P_{2}(\mathbf{k} \cdot \mathbf{a})\right]\left(R_{0}^{-4}-L^{-4}\right)+\frac{\pi L^{3}}{9} \Delta I P_{2}(\mathbf{k} \cdot \mathbf{a})\left(R_{0}^{-6}-L^{-6}\right),
\end{gathered}
$$

For a particle very close to the block boundary, $L<r_{0}$, one can write:

$$
\begin{aligned}
U_{M F}^{(2)}(\mathbf{a}, L) & =-\int_{r_{0}}^{R_{0}} r^{-4} d r \int_{0}^{L / r} d \cos \theta \int_{0}^{2 \pi} d \phi\left[\Delta J+\Delta I(\mathbf{a} \cdot \mathbf{u})^{2}\right]= \\
= & -2 \pi \int_{r_{0}}^{R_{0}} r^{-4} d r \int_{0}^{L / r} d \cos \theta\left[\Delta J+\frac{1}{2} \Delta I \sin ^{2} \theta+\Delta I P_{2}(\cos \theta)(\mathbf{k} \cdot \mathbf{a})^{2}\right]= \\
= & -2 \pi\left(\Delta J+\frac{\Delta I}{3}\right) \int_{r_{0}}^{R_{0}} d r \frac{L}{r^{5}}-\frac{2 \pi}{3} \Delta I P_{2}(\mathbf{k} \cdot \mathbf{a}) \int_{r_{0}}^{R_{0}} d r\left[\frac{L^{3}}{r^{7}}-\frac{L}{r^{5}}\right]= \\
= & \frac{\pi L}{2}\left[\Delta J+\frac{\Delta I}{3}-\frac{\Delta I}{3} P_{2}(\mathbf{k} \cdot \mathbf{a})\right]\left(R_{0}^{-4}-r_{0}^{-4}\right)+\frac{\pi L^{3}}{9} \Delta I P_{2}(\mathbf{k} \cdot \mathbf{a})\left(R_{0}^{-6}-r_{0}^{-6}\right) .
\end{aligned}
$$

One can check that the obtained expressions (??), (??) and (??) are continuous at $L=r_{0}$ and $L=R_{0}$. They can be easily generalized for a particle inside a block $\mathrm{B}$ with the distance $L$ being negative in such case. Note that for the particle close to the block boundary, the potential (??) is an odd function of $L$ and turns to zero when the particle center is directly at the boundary independently of the particle orientation.

Thus we have obtained the analytical expression for the distribution of nano-particles in such diblock copolymer. The orientational order parameter profile can now be obtained by a numerical integration in Eq. (??). Representative nanoparticle density and nematic order parameter profiles are shown in Figs. ?? and ??. 
One can readily see that the nano-particle density profile is only weakly dependent on the strength of the anisotropic interaction (see Fig. ??), and the nano-particles are predominantly located in the block A which is characterized by the strongest interaction between nano-particles and monomers. The nano-particles are orientationally ordered only in the interfacial region on both sides of the boundary. As seen in Fig. ??, the long axes of the nano-particles are aligned parallel and perpendicular to the block boundary on different sides of the boundary, and the nematic order parameter profiles are nearly antisymmetric.

\section{LAMELLAR PHASE. WEAK SEGREGATION}

At any point in an incompressible copolymer, the local densities $\rho_{A}(\mathbf{r})$ and $\rho_{B}(\mathbf{r})$ obey the relation

$$
\frac{\rho_{A}(\mathbf{r})}{\rho_{A 0}}+\frac{\rho_{B}(\mathbf{r})}{\rho_{B 0}}=1
$$

where the densities of the monomers $\mathrm{A}$ and $\mathrm{B}$ in the corresponding pure polymers, $\rho_{A 0}$ and $\rho_{B 0}$, are introduced (see e.g. [?]).

Weak segregation can be described by a single spatial harmonic of the density modulation and then the distributions of monomers $\mathrm{A}$ and $\mathrm{B}$ are given by:

$$
\rho_{A}(\mathbf{r})=\rho_{A 0}[1-\delta \cos (\mathbf{q} \cdot \mathbf{r})], \quad \rho_{B}(\mathbf{r})=\rho_{B 0} \delta \cos (\mathbf{q} \cdot \mathbf{r})
$$

where the modulation wavevector is $\mathbf{q}=2 \pi \mathbf{k} / d$ and where $d$ is the period.

Substituting Eq. (??) into the mean-field potential (??), and using the identity $\cos \left(\mathbf{q} \cdot \mathbf{r}_{l}\right)=$ $\cos \left(\mathbf{q} \cdot \mathbf{r}_{i l}\right) \cos \left(\mathbf{q} \cdot \mathbf{r}_{i}\right)+\sin \left(\mathbf{q} \cdot \mathbf{r}_{i l}\right) \sin \left(\mathbf{q} \cdot \mathbf{r}_{i}\right)$ one obtains:

$$
U_{M F}\left(\mathbf{a}_{i}, \mathbf{r}_{i}\right)=\delta \cos \left(\mathbf{q} \cdot \mathbf{r}_{i}\right) \int d V\left[\Delta J(r)+\Delta I(r)\left(\mathbf{a}_{i} \cdot \mathbf{u}\right)^{2}\right] \cos (\mathbf{q} \cdot \mathbf{r})
$$

where a constant contribution is neglected. Introducing the interaction parameters

$$
\begin{gathered}
\Delta J_{q}=2 \pi \int r^{2} d r \int d \cos \theta\left[\Delta J(r)+\frac{1}{2} \Delta I(r) \sin ^{2} \theta\right] \cos (q r \cos \theta), \\
\Delta I_{q}=2 \pi \int r^{2} d r \Delta I(r) \int d \cos \theta P_{2}(\cos \theta) \cos (q r \cos \theta),
\end{gathered}
$$

one can write

$$
U_{M F}\left(\mathbf{a}_{i}, \mathbf{r}_{i}\right)=\delta \cos \left(\mathbf{q} \cdot \mathbf{r}_{i}\right)\left[\Delta J_{q}+\Delta I_{q}\left(\mathbf{a}_{i} \cdot \mathbf{k}\right)^{2}\right]
$$



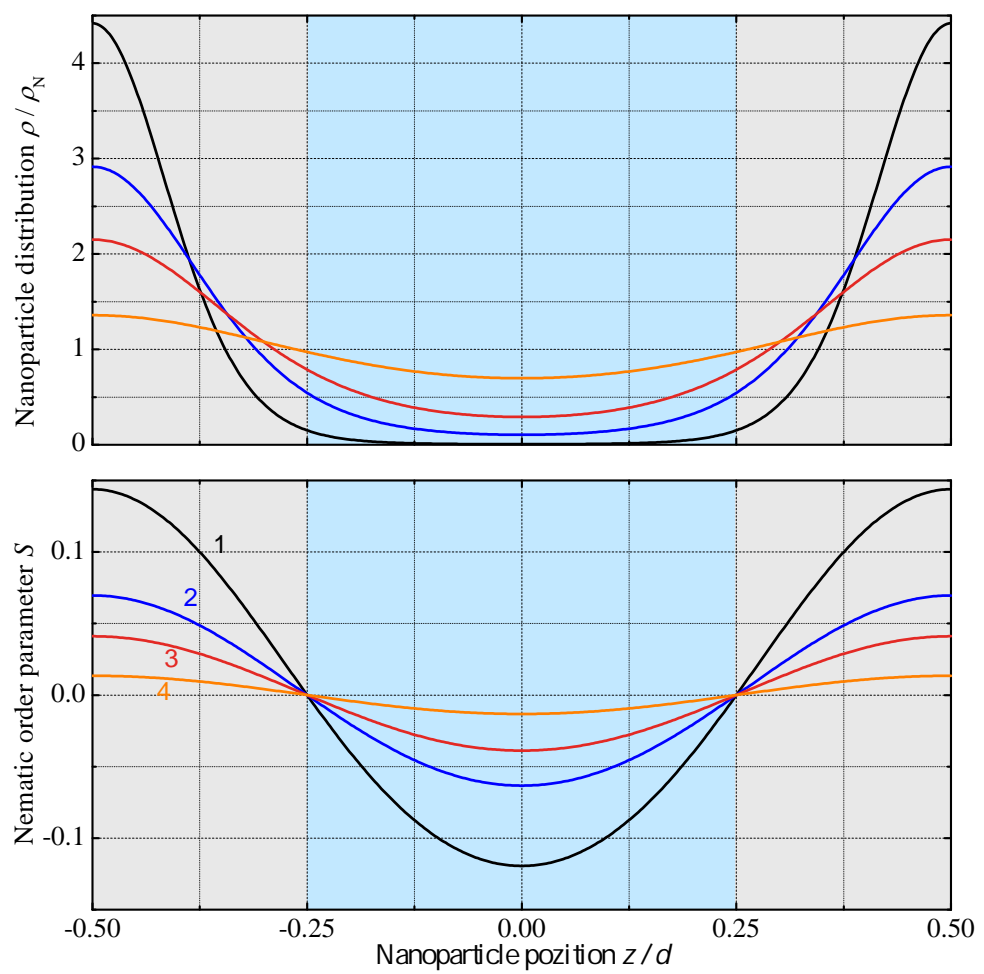

FIG. 2: Nanoparticle local density and nematic order parameter inside a lamellar diblock copolymer with weak segregation. The coordinate $z$ is normalized by the structure period $d$, the isotropic interaction constant is $\Delta J_{q}=3 k_{B} T$ and the anisotropic constant is $\Delta I_{q}=k_{B} T$, and the density spatial modulation is varied as $\delta=1,0.5,0.3,0.1$ for the lines from 1 to 4 respectively.

where for given model interaction potentials, the parameters (??) and (??) can be evaluated numerically. A representative example of thus obtained distributions of the nanoparticle density and nematic order parameter is shown in Fig. ??.

In the important limit when the particle-monomer interactions are short-ranged compared to the lamella block thickness, one can assume $q r \ll 1$ and write

$$
\Delta J_{q} \approx 4 \pi \int\left[\Delta J(r)+\frac{1}{3} \Delta I(r)\right] r^{2} d r
$$

i. e., as independent of $q$, and

$$
\Delta I_{q} \approx-\frac{4 \pi}{15} q^{2} \int \Delta I(r) r^{4} d r
$$

The latter parameter induces the orientational ordering of the nanoparticles and, apparently, it can be estimated as $\left(r_{0} / d\right)^{2}$ times smaller than the parameter $\Delta J_{q}$ which is responsible for the positional segregation of nanoparticles between different blocks. 
One can readily see in Fig. ?? that the nanoparticles are distributed periodically following the distributions of he monomers. At the sme time the nano-particle concentration profiles contain large higher order terms in the Fourier expansion which is determined by the fact that the nano-particles interact stronger with one type of monomers. In contrast, the nematic order parameter profiles are dominated by the first Fourier harmonic.

\section{DISCUSSION}

In this paper nano-particle concentration profiles and the nematic order parameter profiles of anisotropic particles have been calculated in the lamellae and hexagonal phases in the limit of strong segregation taking into account both isotropic and anisotropic interactions between the nano-particles and the monomers of the block copolymer. One notes that it has been possible to derive an analytical expression for the distribution of nano-particles in the lamellae phase. The translational and orientational distributions of nano-particles have also been calculated analytically in the lamellae phase in the opposite limit of weak segregation.

The calculations have been performed in the molecular field approximation which is justified by the fact that the NPs are sufficiently large and interact simultaneously with many monomers/ As a result the fluctuations of the total interaction energy are expected bo be relatively weak. In contrast, at large concentration of NPs the interparticle correlations may become very important as they may be responsible, for example, for the aggregation of NPs. These effects, however, are beyond the scope of the present paper as we assume that the concentration of NPs is very small. One notes also that the present theory assumes that the NPs are not macroscopici i.e. they should be comparable to the monomer size (although slightly bigger). In this case they can be treated as large molecules and their equilibrium distribution is achieved regardless of the initial state of the composite.

It is possible to obtain a crude estimate of the maximum NP molar fraction when the NPs are still relatively uncorrelated. let us assume for simplicity that the NPs are spherical. Then an interaction between the two NPs is sufficiently weak if the second NP is located outside the shell of 12 spherical cites of the same size around the first NP (these spheres make a close packing). This yields an estimate of the critical molar fraction of NPs $\phi \sim 1 / 13$. In practice the NP molar fraction is sufficiently smaller than this.

If the interaction between a nano-particle and the monomers of the blocks $\mathrm{A}$ and $\mathrm{B}$, 
respectively, is sufficiently large, the nano-particles are mainly located inside the block with the strongest interaction. A small fraction of particles also resides in the boundary region between the blocks.In contrast, the anisotropic interaction between NPs and the monomers is responsible for a number of interesting phenomena in the boundary region.

If a nano-particle is located inside a particular block far away from the boundaries, the anisotropic interaction is averaged out and the orientational order parameter of the anisotropic nano-particles vanishes. At the same time, if a nano-particle is sufficiently close to the boundary between blocksi it may interact simultaneously with monomers of both blocks. The corresponding anisotropic interaction constants are generally different, and this asymmetry induces some orientational order of nano-particles in the boundary region. Moreover, the sign of the nematic order parameter is different in the two blocks, that is the rod0like nsno-particles are expected to align parallel to the interface in one block and perpendicular to the interface in another. Taking into account that the density of nano-particles is different in different blocks close to the interface/ one concludes that there is a nonzero average nematic order in the block copolymer. The corresponding average orientational order parameter is small but it may be sufficient to align the polymer by an external electric or magnetic field if the nasno-particle anisotropy is sufficiently large.these theoretical results are qualitatively confirmed by the experiment. For example, functionalized gold nano-rods are aligned parallel or perpendicular to the axis of the cylindrical domain in the hexagonal phase.

One notes that the inhomogeneous distribution of nano-particles in the lamellae and hexagonal phases as well as the orientational ordering of anisotropic nano-particles in the interfacial region between the blocks generally contributes both to the bulk and to the interfacial free energy of these phases. As a result the nano-particles may effect the relative stability of these phases and to shift the parameters of the corresponding phase transitions. A molecular theory which takes these effects into account is in progres. 


\section{Acknowledgments}

M.A.O and Y.V.K. are grateful to the Russian Science Foundation (project 16-13-10280).

[1] M. Alexandre, P. Dubois, Mater. Sci. Eng. Rev. 28, (2000) 1.

[2] A. C. Balazs, Curr. Opin. Colloid Interface Sci. 4, (2000) 443.

[3] E. P. Giannelis, Appl. Organomet. Chem. 12, (1998) 675.

[4] P. P. Soo, B. Y. Huang, Y. I. Jang, Y. M. Chiang, D. R. Sadoway, A. M. Mayes, J. Electrochem. Soc. 146, (1999) 32.

[5] A. C. Balazs, T. Emrick, T. P. Russell, Science 314 (2006) 1107.

[6] R. Krishnamoorti, R. A. Vaia, J. Polym. Sci., Part B: Polym. Phys. 45, (2007) 3252.

[7] A. J. Crosby and J. Y. Lee, Polym. Rev. 47, (2007) 217.

[8] "Organic nanophotonics. Fundamentals and applications". Yong Shend Zhao, editor. Springer, Heidelberg; New York, Dordreht, London, 2014.

[9] "Nanophysics, Nanophotonics, Surface studies and applications." Olena Fesenko, leonid yatsenko, editors. Springer, Heidelbergi New York, Dordreht, London, 2016.

[10] Chan, V. Z. H.; Hoffman, J.; Lee, V. Y.; Iatrou, H.; Avgeropoulos, A.; Hadjichristidis, N.; Miller, R. D.; Thomas, E. L. Science 1999, 286, 1716

[11] Y. Lin, A. Boker, J. B. He, K. Sill, H. Q. Xiang, C. Abetz, X. F. Li, J. Wang, T. Emrick, S. Long, Q. Wang, A. C. Balazs, T. P. Russell, Nature '434, (2005) 55.

[12] J. J. Chiu, B. J. Kim, E. J. Kramer, D. J. Pine, J. Am. Chem. Soc. 127, (2005) 5036.

[13] S. Park, J. Wang, B. Kim, T. P. Russell, Nano Lett. 8, (2008) 1667.

[14] H. Kang, F. A. Detcheverry, A. N. Mangham, M. P. Stoykovich, K.Ch. Daoulas, R. J. Hamers, M. Muller, J. J. de Pablo, P. F. Nealey, Phys. ReV. Lett. 100, (2008) 148303.

[15] R. B. Thompson, V. V. Ginzburg, M. W. Matsen, A. C. Balazs, Science 292, (2001) 2469.

[16] M. R. Bockstaller, Y. Lapetnikov, S. Margel, E. L. Thomas, Journal of the American Chemical Society 125, (2003) 5276 .

[17] M. R. Bockstaller, R. A. Mickiewicz, E. L. Thomas, Adv. Mater. 17, (2005) 1331.

[18] M. J. A. Hore, R. J. Composto, ACS Nano 4, (2010) 6941.

[19] A. L. Frischknecht, M. J. Hore, J. Ford, R. J. Composto, Macromolecules 46, (2013) 2856. 
[20] M. J. Hore, R. J. Composto, Macromolecules 47, (2014) 875.

[21] Q.-Y. Tang, Y.-Q. Ma, J. Phys. Chem. B 113, (2009) 10117.

[22] B. Rasin, H. Chao, G. Jiang, D. Wang, R. A. Riggleman, R. J. Composto, Soft Matter 12, (2016) 2177.

[23] Z. Shou, G. A. Buxton, A. C. Balazs, Composite Interfaces 10, 343 (2003).

[24] S. Sides, B. Kim, E. Kramer, G. Fredrickson, Phys. Rev. Lett. 96, (2006) 250601.

[25] V. Ganesan, A. Jayaraman, Soft Matter 10, (2014) 13.

[26] H. Qi, B. Kinkead, T. Hegmann, Proc. SPIE. 6911, (2008) 691106.

[27] H. Qi, T. Hegmann, J. Mater. Chem. 16, (2006) 4197.

[28] Y. Shiraishi, N. Toshima, H. Maeds, K.and Yoshikawa, J. Xu, S. Kobayashi, Appl. Phys. Lett. 81, (2002) 2845.

[29] S. Kobayashi, N. Toshima, Information Display 23, (2007) 26.

[30] H. Yoshida, K. Kawamoto, H. Kubo, A. Tsuda, T.and Fujii, S. Kuwabata, M. Ozaki, Adv. Mater. 22, (2010) 622.

[31] S. Kaur, S. P. Singh, A. M. Biradar, A. Choudhary, K. Sreeniva, Appl. Phys. Lett. 91, (2007) 023120.

[32] A. Kumar, J. Prakash, A. M. Mehta, D. S. Biradar, W. Haase, Appl. Phys. Lett. 95, (2009) 023117.

[33] Y. Reznikov, O. Buchnev, O. Tereshchenko, V. Reshetnyak, A. Glushchenko, J. West, Appl. Phys. Lett. 82, (2003) 1917.

[34] S. Wiersma, Nature Physics 4, (2008) 359.

[35] V. Gdovinova, N. Tomasovicova, N. Eber, T. Toth-Katona, V. Zavisova, M. Timko, P. Kopcansky, Liq. Cryst. 41, (2014) 1773.

[36] M. V. Gorkunov, M. A. Osipov, Soft Matt. 7, (2011) 4348.

[37] L. M. Lopatina, J. V. Selinger, Phys. Rev. E. 84, (2011) 041703.

[38] M. V. Gorkunov, G. A. Shandryuk, A. M. Shatalova, I. Y. Kutergina, A. S. Merekalov, Y. V. Kudryavtsev, R. V. Talroze, M. A. Osipov, Soft Matt. 9, (2013) 3578.

[39] M. A. Osipov, M. V. Gorkunov, ChemPhysChem 15, (2014) 1496.

[40] M. A. Osipov, M. V. Gorkunov, Phys. Rev. E. 92, (2015) 032501.

[41] B. W. Van der Meer, G. Vertogen in Molecular Physics of Liquid Crystals (Eds.: G. R. Luckhurst, G. W. Gray), Academic Press, New York, 1979. 
[42] M. V. Gorkunov, M. A. Osipov, J. P. F. Lagerwall, and F. Giesselmann, Phys. Rev. E 76, $051706(2007)$

[43] M.A.Osipov, "Molecular theories of liquid crystals", in Handbook of Liquid Crystals: Vol.1. Fundamentals of Liquid Crystals. Goodby, J. W., Collins, P. J., Kato, T., Tschierske, C., Gleeson, H. F. , Raynes, P. (eds.). Weinheim, Germany: Wiley-VCH Verlag GmbH Co. KGaA, Vol. 1, p. 115-168, (2014)

[44] R. D. Deshmukh, Y. Liu, R. J. Composto, NanoLett. 7, (2007) 3662.

[45] E. Ploshnik, A. Salant, U. Banin, R. Shenhar, Adv. Mater. 22, (2010) 2774.

[46] E. Ploshnik, A. Salant, U. Banin, R. Shenhar, Phys. Chem. Chem. Phys. 12, (2010) 11885.

[47] K. Thorkelsson, A. J. Mastroianni, P. Ercius, and T. Xu, NanoLett. 12, (2012) 498.

[48] L. Leibler, Macromolecules 13, (1980) 1602.

Contact details:

M.A.Osipov. E-mail: m.osipov@strath.ac.uk, Tel: +44 1415483655

M.V.Gorkunov. E-mail: gorkunov@ns.crys.ras.ru

Y.V.Kudryavtsev/ E-mail: yar@ips.ac.ru 